\title{
Shape Measures for Triangles
}

\author{
Gerald Farin \\ PRISM/Computer Science and Engineering \\ Arizona State University \\ Tempe, AZ 85287-8809 \\ email farin@asu.edu
}

September 24, 2010

keywords: shape, triangle, condition number, circumellipse

\begin{abstract}
We compare a variety of triangle shape measures using concepts such as smoothness and convexity. We show that one of these measures, the elongation measure, lends itself to an intuitive geometric interpretation.
\end{abstract}

\section{Introduction}

In the context of $2 \mathrm{D}$ triangulations, it is important to obtain "optimal" triangulations, typically in a finite element or scattered data interpolation environment, see [1], [6], [9], [10], [12]. In both applications, one wants to avoid "slivers", i.e., triangles which are either very obtuse or very acute. ${ }^{1}$ These notions can be made more precise by the introduction of exact shape measures. Many such shape measures exist for triangles, all formalizing (in different ways) the no-

\footnotetext{
${ }^{1}$ This is not the case in situations with anisotropic conditions [4].
}

tion of being close to equilateral. We will consider (with $\mathbf{T}$ denoting a triangle)

- maxangle(T): T's largest angle,

- minangle(T): T's smallest angle,

- aspectratio( $\mathbf{T})$ : ratio of radius of $\mathbf{T}$ 's circumcircle to that of its incircle,

- elongation(T): the elongation of T's Steiner circumellipse,

- inverse - mean - ratio(T): an eigenvalue-based measure of T's deviation from being equilateral.

Our approach will be mostly experimental: we will graphically compare the different measures.

One application of these measures is in forming triangulations of $2 \mathrm{D}$ point sets. Using maxangle, we try to find the triangulation which minimizes the largest angle among all of its triangles. The resulting triangulation is the minmax triangulation. Using minangle, we try to find the triangulation which maximizes the smallest angle among all of its triangles. The resulting triangulation is the maxmin triangulation, also known as the 
Delaunay triangulation. The measure aspectratio is mostly used for characterizing finite element meshes.

\section{Shape Criteria}

A measure $s(\mathbf{T})$ for the shape of a triangle $\mathbf{T}$ should have the following properties:

1. $s(\mathbf{T})$ is smallest for equilateral triangles. Motivation: There seems to be general agreement that equilateral triangles are best-shaped.

2. $s(\mathbf{T})$ does not change if $\mathbf{T}$ is scaled uniformly in $x$ and $y$ or if $\mathbf{T}$ is rotated around the origin, i.e., T's centroid. Motivation: Shape should not depend on scale or orientation.

3. $s(\mathbf{T})$ is infinite for degenerate triangles (meaning all $\mathbf{p}_{i}$ are on one straight line). Motivation: There seems to be general agreement that degenerate triangles are worstshaped.

4. $s(\mathbf{T})$ generalizes to simplices in higher dimensions. Motivation: Dimension independence is a mathematically desirable property.

5. $s(\mathbf{T})$ is a convex function. Motivation: A shape measure should not be an undulating function.

\section{The elongation Mea- sure}

A triangle is given by three $2 \mathrm{D}$ points $\mathbf{p}_{1}, \mathbf{p}_{2}, \mathbf{p}_{3}$ whose centroid is the origin (without loss of generality). We represent it by the $2 \times 2$ matrix

$$
\mathbf{T}=\left[\mathbf{p}_{1}, \mathbf{p}_{2}\right] \text {. }
$$

Here, $\mathbf{p}_{1}$ and $\mathbf{p}_{2}$ are interpreted as vectors; the third one, $\mathbf{p}_{3}$, is implied by the centroid condition. See Fig. 1.
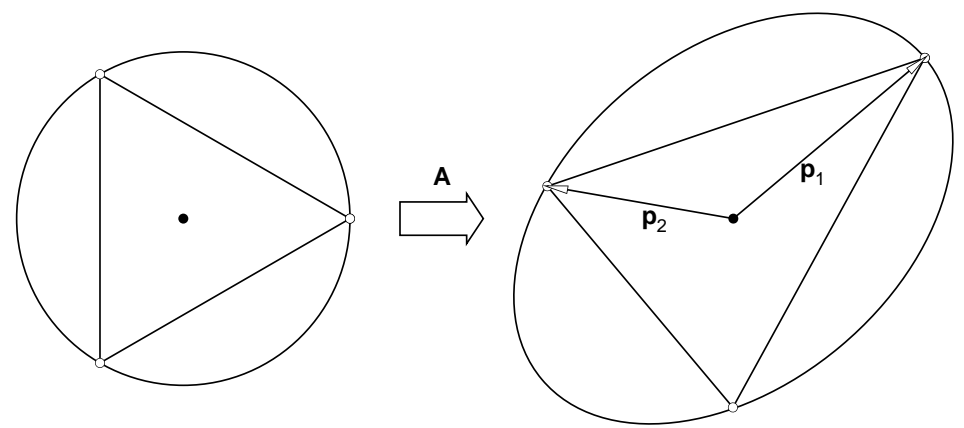

Figure 1: Left: Equilateral triangle and circumcircle. Right: resulting configuration after applying an affine map $A$ (see below).

In the sequel, we will drop the distinction between the triangle and its matrix representation, referring to $\mathbf{T}$ for both of them.

The following triangle shape measure was first introduced by P. Knupp [7], [8].

The matrix

$$
\mathbf{U}=\left[\begin{array}{cc}
1 & -1 / 2 \\
0 & \sqrt{3} / 2
\end{array}\right]
$$

determines an equilateral triangle. Any triangle $\mathbf{T}$ is obtained by applying a linear map $A$ to $\mathbf{U}$ :

$$
\mathbf{T}=A \mathbf{U}
$$

thus $A=\mathbf{T U}^{-1}$.

The singular values $\sigma_{1}$ and $\sigma_{2}$ of $A$ are the square roots of $A^{\mathrm{T}} A$ 's (real and positive) eigenvalues [5]. The ratio $\sigma_{1} / \sigma_{2}$ (assuming that $\sigma_{1} \geq \sigma_{2}$ ) is known as $A^{\prime}$ s condition number; it measures how much 
the map $A$ distorts. We use it to describe T's shape as well:

$$
\operatorname{cond}(\mathbf{T})=\frac{\sigma_{1}}{\sigma_{2}} .
$$

For equilateral triangles, $\operatorname{cond}(\mathbf{T})=1$. For all others, cond $(\mathbf{T})>1$.

A geometric interpretation is as follows. The orthonormal eigenvectors of $A^{\mathrm{T}} A$ may be scaled by $\sigma_{1}$ and $\sigma_{2}$, respectively, and they may then be interpreted as the major and minor axes of an ellipse with elongation

$$
\epsilon(\mathbf{T})=\operatorname{cond}(\mathbf{T})=\sigma_{1} / \sigma_{2},
$$

centered at the origin. This ellipse is circumscribed to $\mathbf{T}$ and is known as T's Steiner circumellipse, see [3]. Among all of T's circumellipses, it has the smallest area.

Because of this intuitive geometric property, we refer to this shape measure as the triangle elongation.

We now discuss how the objectives 15 above are met by the elongation measure.

1. $\epsilon(\mathbf{T})$ is lowest for equilateral triangles. As mentioned above, this criterion is met by design of the shape measure.

2. $\epsilon(\mathbf{T})$ does not change if $\mathbf{T}$ is scaled uniformly in $x$ and $y$. If $\mathbf{T}$ is scaled by a factor $c$, then so is $A$ and hence its eigenvalues. Thus their ratio remains constant.

$\epsilon(\mathbf{T})$ does not change if $\mathbf{T}$ is rotated around the origin, i.e., T's centroid. If $\mathbf{T}$ is rotated by a matrix $R$, the matrix $A$ is changed to $A^{\prime}=R \mathbf{T} \mathbf{U}^{-1}$, and thus $A^{\mathrm{T}} A=A^{\prime \mathrm{T}} A^{\prime}$.

3. $\epsilon(\mathbf{T})$ is infinite for degenerate triangles (meaning all $\mathbf{p}_{i}$ are on one straight line). A general $\mathbf{T}$ has rank 2, whereas a degenerate one only has rank 1 , resulting in $A$ being singular. Thus, $\epsilon(\mathbf{T})=\infty$.
4. $\epsilon(\mathbf{T})$ 's definition can be applied verbatim to simplices in any dimension.

5. $\epsilon(\mathbf{T})$ is convex as outlined in the next paragraph.

Without loss of generality, we may assume that one edge of the triangle $\mathbf{T}$ is the line segment connecting the two points $(-1,0)$ and $(1,0)$. Let the third point have coordinates $(x, y)$. As $x$ and $y$ vary, the resulting triangle $\mathbf{T}(x, y)$ will have different shapes $\epsilon(\mathbf{T}(x, y))$.

It is easy to see (using Mathematica) that

$$
\epsilon(\mathbf{T}(x, y))=\sqrt{\frac{a+b}{a-b}}
$$

where $a=3+x^{2}+y^{2}$ and $b=$ $\sqrt{x^{4}+\left(y^{2}-3\right)^{2}+2 x^{2}\left(y^{2}+3\right)}$. We can also verify that all contours of the form

$$
\epsilon(\mathbf{T}(x, y))=c
$$

are circles, having centers $\mathbf{c}(c)$ and radii $r(c)$ :

$\mathbf{c}(c)=\frac{\sqrt{3}}{2 c}\left[\begin{array}{c}0 \\ \left(c^{2}+1\right)\end{array}\right], \quad r(c)=\frac{\sqrt{3}}{2 c}\left(c^{2}-1\right)$.

The determinant of $\epsilon(\mathbf{T}(x, y))$ 's Hessian has a minimum at $(0,2)$ and is nonnegative everywhere (symbolic computation using Mathematica). Hence $\epsilon(\mathbf{T}(x, y))$ is a convex bivariate function. Its minimum is at $(x, y)=(0, \sqrt{3})$, corresponding to the vertex of an equilateral triangle. At this point, $\epsilon(\mathbf{T}(x, y))$ is not differentiable.

\section{Comparison of Shape Measures}

We graphically compare the above shape measures. The shape measure for the triangle $\mathbf{T}(x, y)$ is plotted as a $z$-value at $(x, y)$. This results in a high degree of 
symmetry in the plots. The points ($1,0)$ and $(1,0)$ are not marked in the plots but their position should be identifiable through the axis labeling.

\subsection{Maximum Angle}

Traditionally, the smallest or the largest angle are chosen as shape measures, in particular when constructing triangulations. Figure 2 shows the behavior of the measure "maximum angle". Notice that maxangle( $\mathbf{T}(x, y))$ is not a smooth function. ${ }^{2}$ This is because as $x$ and $y$ vary, the attribute of being the maximal angle will jump from one vertex angle to another. This shape measure satisfies properties 1 and 2 of section 2 .

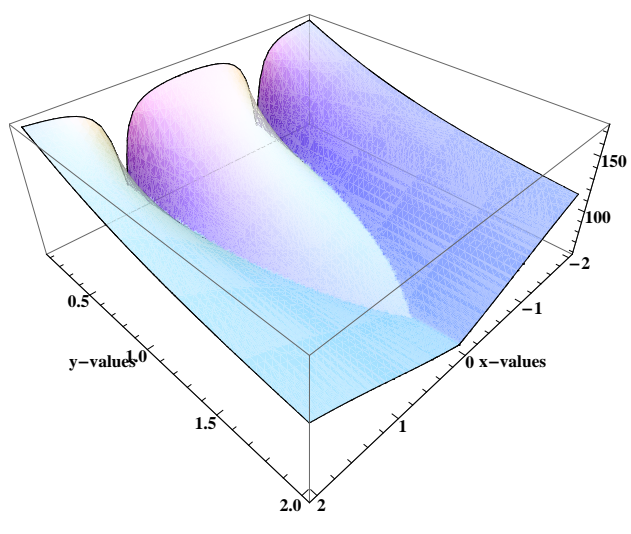

Figure 2: Triangle shape: the values of maxangle $(\mathbf{T}(x, y))$ over the domain $-2 \leq x \leq 2,0.01 \leq y \leq 2$. Vertical axis: maxangle

\subsection{Minimum Angle}

The minimum angle in a triangle leads is widely used as a shape measure when

\footnotetext{
${ }^{2}$ Near the maximum values of maxangle, truncation happens. This also applies to subsequent plots.
}

constructing triangulations. Maximizing the minimal angle in a triangulation leads to the Delaunay triangulation. This shape measure satisfies property 2 of section 2 .

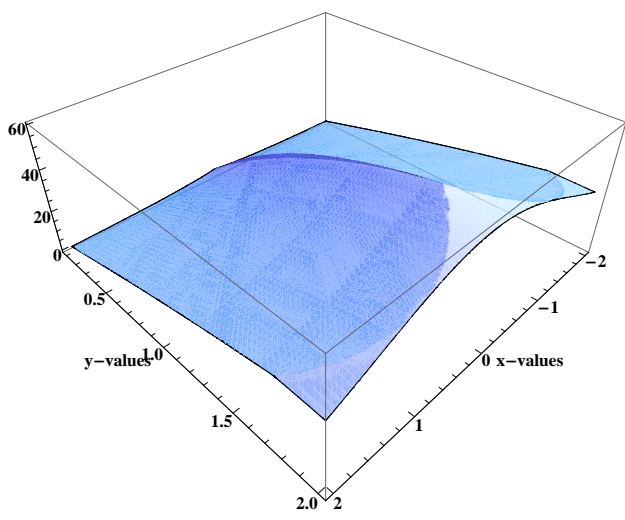

Figure 3: Triangle shape: the values of minangle $(\mathbf{T}(x, y))$ over the domain $-2 \leq$ $x \leq 2,0.01 \leq y \leq 2$. Vertical axis: minangle

\subsection{Max/Min Ratio}

Neither the minangle nor the maxangle measures satisfy requirement 3 of Section 2. By using their ratio, we have a measure satisfying requirements $1,2,3,4$. The corresponding plot is shown in Fig. 4 and it is, as expected, not convex, violating requirement 5 .

The plot (and others) is clipped in the $z$-direction in order to avoid problems with very large $z$-values.

\subsection{Aspect Ratio}

The aspect ratio: ratio of circumradius over inradius is used in finite elements. This function is plotted in Fig. 5. It satisfies requirements $1,2,3,4$. 


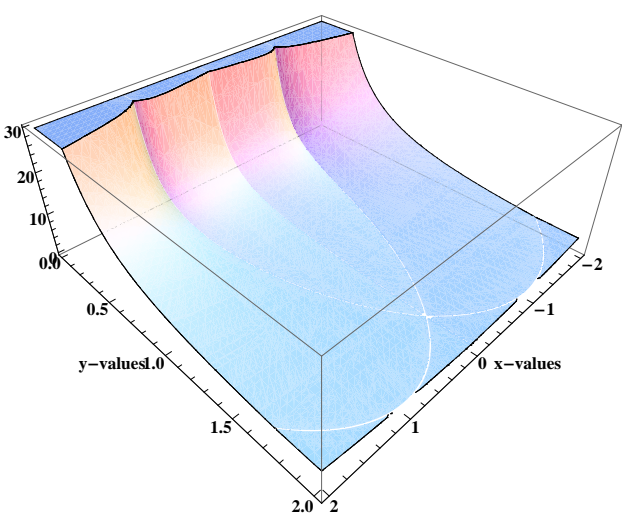

Figure 4: Triangle shape: the values of maxangle $\mathbf{T}(x, y) /$ minangle $\mathbf{T}(x, y)$ over the domain $-2 \leq x \leq 2,0.01 \leq y \leq$ 2.

\subsection{Inverse Mean Ratio}

This inverse mean ratio measure $\rho(\mathbf{T})$ is given by (using notation of section 3 )

$$
\rho(\mathbf{T})=\frac{\sigma_{1}^{2}+\sigma_{2}^{2}}{2 \sigma_{1} \sigma_{2}} .
$$

It is described by T. Munson [11] and goes back to Knupp [8], [7]. As a function of a variable vertex $(x, y)$ (compare to eq. (1)), it may be written as

$\rho(\mathbf{T}(x, y))=\frac{81+x^{4}+y^{4}+2 x^{2}\left(y^{2}+9\right)}{9 y^{2}}$

and is plotted in Fig. 6. This function is smooth everywhere (except at $y=0$ ) and convex (as shown in [11]). It satisfies all requirements of section 2 .

Some of its contours are shown in Fig. 7 .

\subsection{Triangle elongation}

Finally, we plot the shape measure $\epsilon(\mathbf{T}(x, y))$. The corresponding plot is

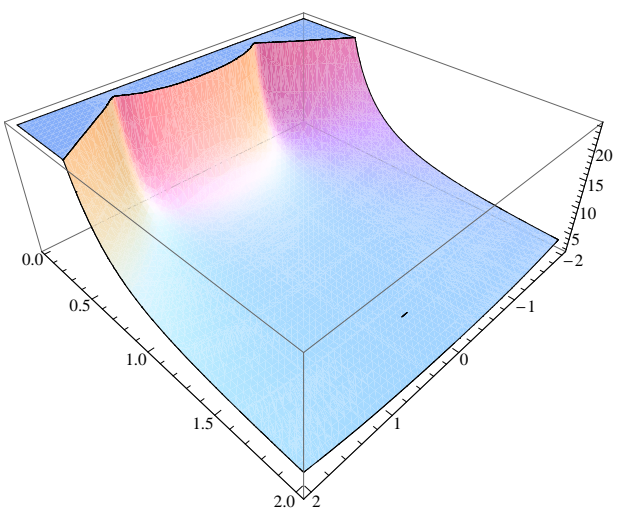

Figure 5: Triangle shape: the values of aspectratio $\mathbf{T}(x, y)$ over the domain $-2 \leq x \leq 2,0.01 \leq y \leq 2$.

shown in Fig. 8. Its contours are shown in Fig. 9.

Note that while the plots of $\rho(x, y)$ and $\epsilon(x, y)$ are hardly distinguishable, their contours have significantly different shape, raising the question if any practical implications (such as in mesh optimization or scattered data interpolation) arise from this.

\section{Conclusion and Out- look}

We investigated the elongation measure for describing the shape of triangles. It is a smoother measure (by visual inspection) than traditional ones which we used as a comparison. This raises the question if increased smoothness of a shape measure has practical benefits.

The basic concept of the elongation measure applies to polygons as well - all we have to do is replace the $2 \times 2$ matrix $\mathbf{T}$ by a $2 \times n$ matrix $\mathbf{P}$. We have successfully compared shapes of polygons with 


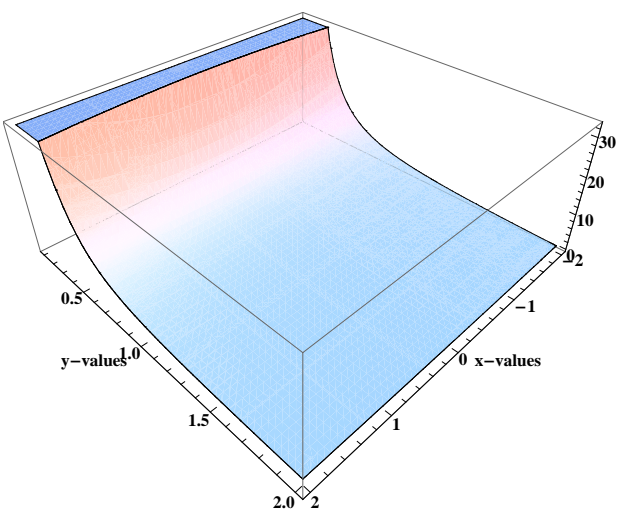

Figure 6: Triangle shape: the values of $\rho(\mathbf{T}(x, y))$ over the domain $-2 \leq x \leq$ $2,0.01 \leq y \leq 2$.

different numbers of vertices [2]. But since the proposed measure is blind to the ordering of polygon vertices, more work is necessary for the case of polygons.

The extension of the elongation measure to higher dimensions (tetrahedra and simplices) is straightforward.The author conjectures that the important concept of convexity holds for higher dimensions as well.

\section{References}

[1] I. Babuska and A. Aziz. On the angle condition in the finite element method. SIAM J Numer. Analysis, 13(2):214-227, 1976.

[2] A. Constantiniu, P. Steinmann, T. Bobach, G. Farin, and H. Hagen. The Adaptive Delaunay Tessellation: a Neighborhood Covering Meshing Technique. Computational Mechanics, 42:655-669, 2008.

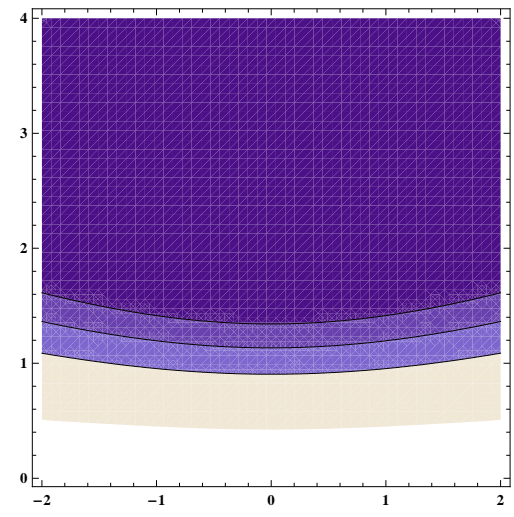

Figure 7: Contours of $\rho(\mathbf{T}(x, y))$.

[3] H. Doerrie, D. Antin. 100 great problems of elementary mathematics: their history and solution. Dover, 1965

[4] J. Dompierre, M.-G. Vallet, P. Labbe, F. Guibault. An analysis of simplex shape measures for anisotropic meshes. Computer Methods in Applied Mechanics and Engineering, 194(48-49):4896-4914, 2005.

[5] G. Farin and D. Hansford. Mathematical Principles for Scientific Computing and Visualization. AK Peters, 2008.

[6] D. Field. Qualitative measures for initial meshes. Int. J. Num. Methods in Engineering, 47:709-712, 2000.

[7] L. Freitag and P. Knupp. Tetrahedral Mesh Improvement via Optimization of the Element Condition Number. Int. J. Num. Methods in Engineering, 53(6):1377-1391, 2001.

[8] P. Knupp. Algebraic Mesh Quality Metrics. SIAM J. Scientific Computing, 23: 193-218, 2001. 


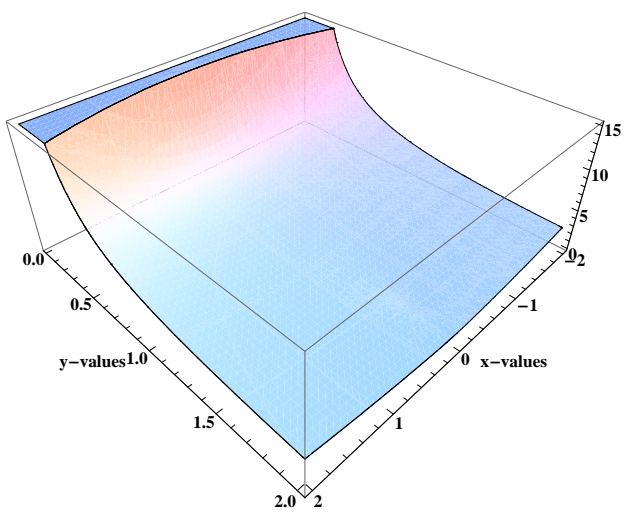

Figure 8: Triangle shape: the values of $\epsilon(\mathbf{T}(x, y))$ over the domain $-2 \leq x \leq$ $2,0.01 \leq y \leq 2$.

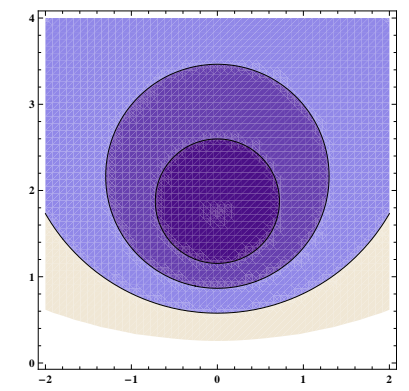

Figure 9: Contours of $\epsilon(\mathbf{T}(x, y))$.

[9] C. Lawson. Transforming triangulations. Discrete Mathematics, 3:365$372,1971$.

[10] D. Mackenzie. What is the Shape of a Triangle? Note di Mathematica, 13: 237-250, 1993.

[11] T. Munson. Mesh shape-quality optimization using the inverse meanratio metric. Math. Program. Ser. A 110:561-590, 2007

[12] J. Shewchuk. What is a good linear element? interpolation, condi- tioning, and quality measures. In Proc. of the 11th International Meshing Roundtable, pages 115-126, 2002. 